\title{
The Higher Order Crack Tip Fields for Anti-plane Crack in Power Functionally Graded Piezoelectric Materials
}

\author{
Dai Yao ${ }^{1, a}$, Chong Xiao ${ }^{1, b}$ Pan Jing-wen ${ }^{1, \text { a }}$ \\ ${ }^{1}$ The Academy of Armored Force Engineering, Beijing, 100072 \\ adai_yao@sina.com, ${ }^{\text {b}}$ chongxiao2005@163.com
}

Keywords: higher order fields, power FGPMs, eigen-expansion method, anti-plane crack

\begin{abstract}
The eigen-solutions of the crack tip fields for an anti-plane crack in functionally graded piezoelectric materials (FGPMs) are studied. Different from previous analyses, all material properties of the FGPM are assumed to be power-law function of $y$ perpendicular to the crack. The crack surfaces are electrically impermeable and loaded by anti-plane shear tractions and in-plane electric displacements. The higher order crack tip fields of FGPMs are obtained by the eigen-expansion method. The stress intensity factor and electric displacement intensity factor are obtained explicitly.
\end{abstract}

\section{Introduction}

Piezoelectric materials have been widely used in modern technology due to their electromechanical coupling behavior. To improve the reliability of the modern structures, the concept of FGPMs has been introduced recently. Due to their brittleness, the fracture problems of FGPMs has been attracted extensive attention in order to meet the demand of high strength and high temperature applications. Ou [1] studied the internal crack problem located within one functionally graded piezoelectric strip. The crack is normal to the edge of the strip and the material properties vary along the direction of crack length. Kwon [2] analyzed the electrical nonlinear behavior of an anti-plane shear crack in a functionally graded piezoelectric strip by using the strip saturation model within the framework of linear electro-elasticity. Hsu [3] investigated the fracture behavior of an arbitrarily oriented crack in a FGPM subjected to anti-plane mechanical and in-plane electric loads. Zhang [4] studied the behavior of four parallel non-symmetric permeable cracks with different lengths in a FGPM plane subjected to anti-plane shear stress loading by the Schmidt method. To our knowledge, the electro-elastic behavior of a crack in FGPMs with power gradation has not been studied in the open literatures. It is with this in mind that we report the present work. In the present paper, we attempt to obtain the higher order mechanical and electric crack tip fields by eigenexpansion method.

\section{Basic equations}

Consider a crack of length $2 L$ in FGPM planes, as shown in Fig.1. The FGPM is poled in the $z$ direction and isotropic in the xoy plane. It is loaded by anti-plane shear tractions and in-plane electric displacements, the crack is electrically impermeable and the crack surfaces are traction free. We assume that the material properties are represented by the following case

$$
c_{44}=c_{440}(1+\lambda y)^{k}, \quad e_{15}=e_{150}(1+\lambda y)^{k}, \quad \varepsilon_{11}=\varepsilon_{110}(1+\lambda y)^{k}
$$

where $c_{44}$ is the shear modulus, $e_{150}$ is the piezoelectric coefficient, $\varepsilon_{110}$ is the dielectric parameter at $y=0$. 


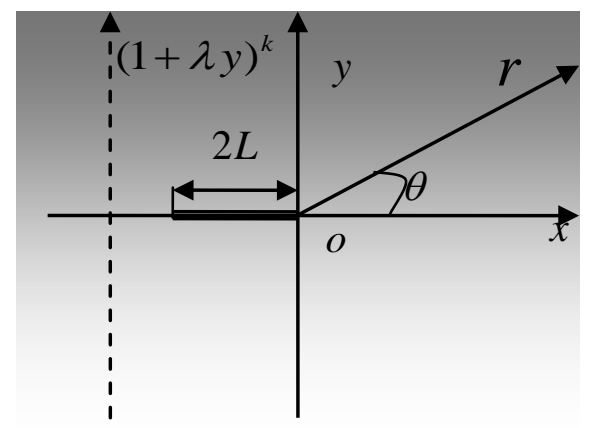

Fig.1Anti-plane crack in FGPMs

The governing equations can be written as

$$
\begin{aligned}
& \nabla^{2} w+\frac{k \lambda}{1+\lambda r \sin \theta}\left(\sin \theta \frac{\partial w}{\partial r}+\frac{1}{r} \cos \theta \frac{\partial w}{\partial \theta}\right)=0 \\
& \nabla^{2} \phi+\frac{k \lambda}{1+\lambda r \sin \theta}\left(\sin \theta \frac{\partial \phi}{\partial r}+\frac{1}{r} \cos \theta \frac{\partial \phi}{\partial \theta}\right)=0
\end{aligned}
$$

where $\nabla^{2}=\frac{\partial^{2}}{\partial r^{2}}+\frac{1}{r} \frac{\partial}{\partial r}+\frac{1}{r^{2}} \frac{\partial^{2}}{\partial \theta^{2}}$ is the two-dimensional Laplace operator.

\section{The higher order crack-tip fields}

The displacement component $w$ and the electric potential $\phi$ can be expanded as follows

$$
w=\sum_{i=1}^{\infty} r^{\frac{i}{2}} w_{i}(\theta), \quad \phi=\sum_{i=1}^{\infty} r^{\frac{i}{2}} \phi_{i}(\theta)
$$

where, $w_{i}(\theta)$ and $\phi_{i}(\theta)$ are eigen-functions.

Substitute Eq.(3) into Eq.(2). According to the linear independence of $r^{-3 / 2}, r^{-1}$, $r^{-1 / 2}, \ldots, r^{i / 2-2}, \ldots$, the system of ordinary differential equations are obtained.

$$
\left\{\begin{array}{l}
\frac{1}{4} \mathbf{w}_{1}(\theta)+\frac{\partial^{2} \mathbf{w}_{1}}{\partial \theta^{2}}=0 \\
\mathbf{w}_{2}(\theta)+\frac{\partial^{2} \mathbf{w}_{2}}{\partial \theta^{2}}=0 \\
\cdots \\
\frac{i^{2}}{4} \mathbf{w}_{i}+\frac{\partial^{2} \mathbf{w}_{i}}{\partial \theta^{2}}+\lambda\left[\frac{(i-2)(i-2+2 k)}{4} \sin \theta \mathbf{w}_{i-2}+k \cos \theta \frac{\partial \mathbf{w}_{i-2}}{\partial \theta}+\sin \theta \frac{\partial^{2} \mathbf{w}_{i-2}}{\partial \theta^{2}}\right]=0 \\
\cdots
\end{array}\right.
$$

where $\mathbf{w}=\left[\begin{array}{l}w \\ \phi\end{array}\right]$.

In the case of electrically impermeable crack, the crack surfaces are free of electric charges and the electric displacement inside the crack is zero. As the crack surface is free, the boundary conditions are

$$
\left.\sigma_{z y}\right|_{\theta= \pm \pi}=0,\left.\quad D_{y}\right|_{\theta= \pm \pi}=0
$$

Further, they can be expressed as

$$
w_{i}^{\prime}( \pm \pi)=0 \quad \phi_{i}^{\prime}( \pm \pi)=0
$$


Solving the system of ordinary differential equations, we can obtain the results

$$
\left\{\begin{array}{l}
w_{1}(\theta)=A_{11} \sin \frac{\theta}{2} \\
w_{2}(\theta)=A_{21} \cos \theta \\
w_{3}(\theta)=A_{31} \sin \frac{3 \theta}{2}-\frac{1}{12} k \lambda A_{11} \cos \frac{3 \theta}{2}-\frac{1}{4} k \lambda A_{11} \cos \frac{\theta}{2} \\
w_{4}(\theta)=A_{41} \cos 2 \theta \\
w_{5}(\theta)=A_{51} \sin \frac{5 \theta}{2}+\frac{1}{20} k \lambda A_{31} \cos \frac{5 \theta}{2}+\frac{k+2}{16} k \lambda^{2} A_{11} \sin \frac{3 \theta}{2}+\frac{k+2}{48} k \lambda^{2} A_{11} \sin \frac{\theta}{2}-\frac{1}{4} k \lambda A_{31} \cos \frac{\theta}{2} \\
w_{6}(\theta)=A_{61} \cos 3 \theta-\frac{1}{12} k \lambda A_{41} \sin 3 \theta+\frac{1}{4} k \lambda A_{41} \sin \theta \\
\ldots \ldots \\
\phi_{1}(\theta)=B_{11} \sin \frac{\theta}{2} \\
\phi_{2}(\theta)=B_{21} \cos \theta \\
\phi_{3}(\theta)=B_{31} \sin \frac{3 \theta}{2}-\frac{1}{12} k \lambda B_{11} \cos \frac{3 \theta}{2}-\frac{1}{4} k \lambda B_{11} \cos \frac{\theta}{2} \\
\phi_{4}(\theta)=B_{41} \cos 2 \theta \\
\phi_{5}(\theta)=B_{51} \sin \frac{5 \theta}{2}+\frac{1}{20} k \lambda B_{31} \cos \frac{5 \theta}{2}+\frac{k+2}{16} k \lambda^{2} B_{11} \sin \frac{3 \theta}{2}+\frac{k+2}{48} k \lambda^{2} B_{11} \sin \frac{\theta}{2}-\frac{1}{4} k \lambda B_{31} \cos \frac{\theta}{2} \\
\phi_{6}(\theta)=B_{61} \cos 3 \theta-\frac{1}{12} k \lambda B_{41} \sin 3 \theta+\frac{1}{4} k \lambda B_{41} \sin \theta
\end{array}\right.
$$

where $A_{i j}$ and $B_{i j}$ are the undetermined coefficients.

Substituting Eq. (7) and (8) into Eq.(3), the displacement component $w$ and the electric potential $\phi$ are obtained.

$$
\begin{aligned}
w= & r^{\frac{1}{2}} A_{11} \sin \frac{\theta}{2}+r A_{21} \cos \theta+r^{\frac{3}{2}}\left(A_{31} \sin \frac{3 \theta}{2}-\frac{1}{12} k \lambda A_{11} \cos \frac{3 \theta}{2}-\frac{1}{4} k \lambda A_{11} \cos \frac{\theta}{2}\right)+r^{2} A_{41} \cos 2 \theta \\
& +r^{\frac{5}{2}}\left(A_{51} \sin \frac{5 \theta}{2}+\frac{1}{20} k \lambda A_{31} \cos \frac{5 \theta}{2}+\frac{k+2}{16} k \lambda^{2} A_{11} \sin \frac{3 \theta}{2}+\frac{k+2}{48} k \lambda^{2} A_{11} \sin \frac{\theta}{2}-\frac{1}{4} k \lambda A_{31} \cos \frac{\theta}{2}\right)+ \\
& r^{3}\left(A_{61} \cos 3 \theta-\frac{1}{12} k \lambda A_{41} \sin 3 \theta+\frac{1}{4} k \lambda A_{41} \sin \theta\right)+\cdots \\
\phi & =r^{\frac{1}{2}} B_{11} \sin \frac{\theta}{2}+r B_{21} \cos \theta+r^{\frac{3}{2}}\left(B_{31} \sin \frac{3 \theta}{2}-\frac{1}{12} k \lambda B_{11} \cos \frac{3 \theta}{2}-\frac{1}{4} k \lambda B_{11} \cos \frac{\theta}{2}\right)+r^{2} B_{41} \cos 2 \theta \\
& +r^{\frac{5}{2}}\left(B_{51} \sin \frac{5 \theta}{2}+\frac{1}{20} k \lambda B_{31} \cos \frac{5 \theta}{2}+\frac{k+2}{16} k \lambda^{2} B_{11} \sin \frac{3 \theta}{2}+\frac{k+2}{48} k \lambda^{2} B_{11} \sin \frac{\theta}{2}-\frac{1}{4} k \lambda B_{31} \cos \frac{\theta}{2}\right)+ \\
& r^{3}\left(B_{61} \cos 3 \theta-\frac{1}{12} k \lambda B_{41} \sin 3 \theta+\frac{1}{4} k \lambda B_{41} \sin \theta\right)+\cdots
\end{aligned}
$$

Then, the stress and the electric displacement components can be obtained

$$
\begin{array}{ll}
\tau_{x z}=c_{44} w_{, x}+e_{15} \phi_{, x}, & \tau_{y z}=c_{44} w_{, y}+e_{15} \phi_{, y} \\
D_{x}=e_{15} w_{, x}-\varepsilon_{11} \phi_{, x}, & D_{y}=e_{15} w_{, y}-\varepsilon_{11} \phi_{, y}
\end{array}
$$

Further, the stress and the electric displacement components can be obtained. The mode III stress intensity factor (SIF) and electric displacement intensity factor (EDIF) of the crack tip are 
defined as

$$
\left\{\begin{array}{l}
K^{T}=\lim _{r \rightarrow 0} \sqrt{2 \pi r} \sigma_{y z}(r, 0)=\frac{\sqrt{2 \pi}}{2}\left(c_{440} A_{11}-e_{150} B_{11}\right) \\
K^{D}=\lim _{r \rightarrow 0} \sqrt{2 \pi r} D_{y}(r, 0)=\frac{\sqrt{2 \pi}}{2}\left(e_{150} A_{11}+\varepsilon_{110} B_{11}\right)
\end{array}\right.
$$

\section{Conclusion}

The higher order mechanical and electric crack-tip fields for power FGPMs are obtained by the methods of eigen-expansion in this paper. The results showed that the non-homogeneous material parameters $k$ and $\lambda$ first appeared in the third order fields, $k^{2}$ and $\lambda^{2}$ in the fifth order ones. It is clear that non-homogeneous material parameters strongly affect the higher order items of the fields. The explicit expression of stress intensity factor and electric displacement intensity factor are presented.

\section{Acknowledgements}

The research is supported by the National Natural Science Foundation of China (No11172332.)

\section{References}

[1] Y.-L. Ou, C.-H. Chue: International Journal of Solids and Structures. Vol.43 (2006), p. 6148

[2] S. M. Kwon: International Journal of Solids and Structures. Vol.40 (2003), p. 5649

[3] W.-H. Hsu, C.-H. Chue: Journal of the Chinese Institute of Engineers. Vol.32 (2009), p. 383

[4] P. W. Zhang, Z. G. Zhou, G. Li: Z. Angew. Math. Mech. Vol.89 (2009), p. 767 